\title{
THEORY AND OBSERVATIONS OF NON-THERMAL PHENOMENA IN HOT MASSIVE BINARIES
}

\author{
RICHARD L. WHITE \\ Space Telescope Science Institute, 3700 San Martin Dr., Baltimore, MD 21218, U.S.A. \\ and \\ WAN CHEN \\ GSFC/USRA, Code 661, Greenbelt, MD 20771, U.S.A.
}

\begin{abstract}
The shock between the colliding winds in binary systems containing two massive stars accelerates particles to relativistic energies. These energetic particles can produce observable non-thermal radiation from the radio to $\gamma$-rays. The important physical processes in such systems are very similar to those we have proposed for non-thermal emissions from single hot stars, which have shocks generated by instabilities in the radiatively driven stellar winds. This paper discusses the theory and observations of non-thermal radiation in the radio, $\mathrm{X}$-ray, and $\gamma$-ray regions from both single stars and massive binaries. Similarities and differences between the two types of systems are outlined. We discuss two important physical effects that apparently have been neglected in previous theoretical work on colliding wind binaries.
\end{abstract}

Key words: stars: massive binaries - colliding winds - individual: HD 193793

\section{Introduction}

The important physical processes in colliding wind binaries are very similar to those that account for non-thermal emissions from single hot stars, which have shocks generated by instabilities in the radiatively driven stellar winds. Both electrons and nuclei are accelerated from thermal to relativistic energies via the first-order Fermi mechanism. The highest energy achieved by protons is determined mainly by the geometry of the system, while the limiting energy for electrons is determined by a balance between shock acceleration and radiative losses via inverse Compton scattering of the copious stellar ultraviolet photons. These inverse Compton-scattered photons emerge with X-ray and $\gamma$-ray energies and typically carry the largest amount of energy of any non-thermal radiation. The relativistic electrons emit radio synchrotron emission with a luminosity that is determined by both the electron energy spectrum and the photospheric magnetic field carried out in the stellar winds. The Razin effect plays an important role in suppressing low frequency radio emission. Collisions between relativistic protons and the thermal population of stellar wind nuclei leads to high-energy $\gamma$-rays from $\pi^{0}$ decays. 


\section{Evidence for non-thermal emissions from single stars}

By far the best evidence for particle acceleration in winds comes from the observation of non-thermal radio emission, which can only be understood as synchrotron emission from relativistic electrons. Abbott, Bieging, \& Churchwell $(1984,1985)$ found that a significant fraction of OB and Wolf-Rayet stars show non-thermal radio emission. This non-thermal emission can be identified either through its unusual spectral index or because it has a surface brightness too high to be the result of thermal emission (White \& Becker 1982; Becker \& White 1985). White (1985) suggested that this could be explained as synchrotron radiation from electrons accelerated to relativistic energies by the same shocks that emit the Einstein X-rays. Since these relativistic particles must be generated very far from the stellar surface (Chen \& White 1994), they must be accelerated by shocks. White

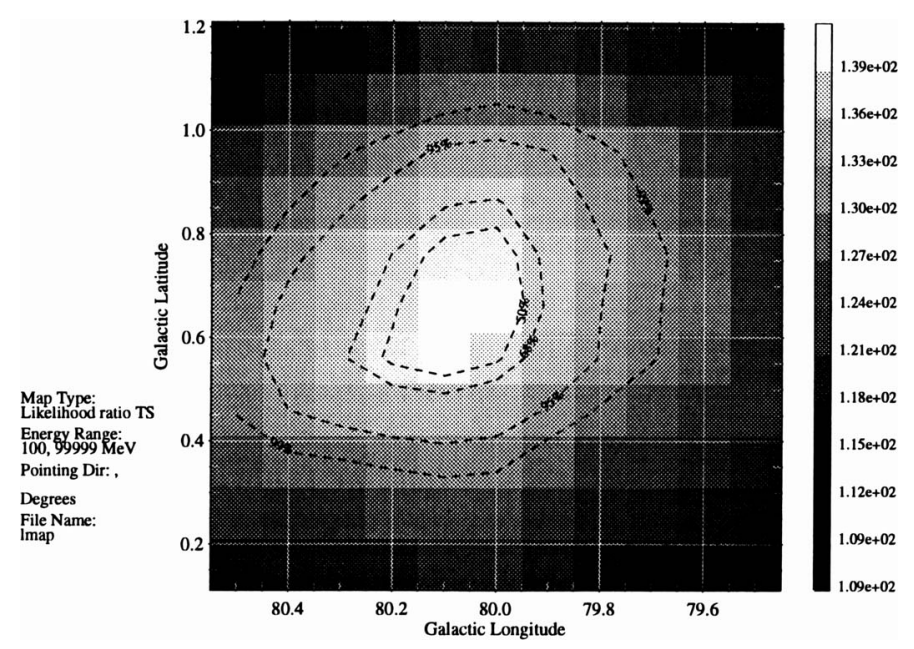

Fig. 1. GRO EGRET map of 0.1-4 GeV $\gamma$-ray emission in the vicinity of the Cyg OB2 cluster of stars. There is definitely a detection, but unfortunately the position is not quite accurate enough to rule out the nearby X-ray binary Cyg X-3 as the source. The Cyg OB2 cluster lies near the center of the emission, while Cyg X-3 is just inside the $95 \%$ confidence boundary to the west.

(1985) also suggested that the relativistic protons accelerated by the shocks should emit $\gamma$-rays via the decay of $\pi^{0}$ particles generated through protonproton collisions. Pollock $(1987,1991)$ argued that the relativistic electrons would produce X-rays through inverse Compton scattering of the stellar UV photons and would produce $\gamma$-rays through the Bremsstrahlung mechanism; Chen \& White $(1991 \mathrm{a}, \mathrm{b})$ computed the X-ray to $\gamma$-ray spectrum from inverse Compton scattering, and Montmerle (1990) also estimated the $\gamma$-ray emission from wind-accelerated particles. 
The observational evidence for the other diagnostics of particle acceleration is marginal or absent at the moment, though this is expected based on the sensitivity of current observations. There is some weak evidence from Einstein SSS spectra for the predicted hard X-rays from inverse Compton scattering (Chen \& White 1991a), though other explanations are possible (Cassinelli \& Swank 1983). Observations by the ASCA X-ray satellite should either confirm or demolish the theory that the hard X-rays are of non-thermal origin. Searches are underway for the predicted $\gamma$-ray emission using the Gamma Ray Observatory, with encouraging but inconclusive results so far (see Fig. 1).

\section{Binaries versus single stars: similarities}

Binary systems consisting of two hot, massive stars, both with stellar winds, can accelerate particles at the shock where the winds collide. The hydrodynamics of such systems have been studied in several papers (Luo, McCray, \& Mac Low 1990; Stevens, Blondin, \& Pollock 1992), and Eichler \& Usov (1993) have discussed both particle acceleration and synchrotron emission from colliding winds.

\subsection{INVERSE COMPTON LOSSES FOR RELATIVISTIC ELECTRONS}

There are many similarities between colliding wind binaries and the singlestar systems discussed above. The shock acceleration mechanism is, of course, the same; less trivially, the most important energy loss mechanism for relativistic electrons is inverse Compton (IC) scattering. IC losses affect the accelerated electrons in two ways. First, in the vicinity of the shock, the competition between IC losses and shock acceleration determines the highest energy to which electrons can be accelerated. If we assume that the electron diffusion length is equal to the gyral radius (which is appropriate when the magnetic field is parallel to the shock front),

$$
E_{m a x}=71 \mathrm{GeV}\left(\frac{v_{s, 8} B}{L_{6}}\right)^{1 / 2} r_{A U}
$$

where $v_{s, 8} 10^{3} \mathrm{~km} / \mathrm{s}$ is the shock velocity, $B$ is the magnetic field in Gauss, $L_{6} 10^{6} L_{\odot}$ is the stellar luminosity, and $r_{A U}$ is the distance from the star in astronomical units.

Second, after the electrons leave the vicinity of the shock they rapidly lose their energy through IC scattering. If gas escapes from the vicinity of the shock at the escape velocity $v_{e s c, 8} 10^{3} \mathrm{~km} / \mathrm{s}$, then after a dynamical time $r / v_{e s c}$ all high energy electrons have cooled to

$$
E_{c}=2.2 \mathrm{MeV} \frac{r_{A U} v_{e s c, 8}}{L_{6}} .
$$


Note that this energy is usually much lower than the maximum energy to which the electrons are accelerated.

For a shock with a compression ratio $\chi$, the electron energy spectrum $d N / d p$ generated by shock acceleration with IC losses is (Chen 1992)

$$
\frac{d N}{d p}=\frac{N_{0}}{p_{0}}\left(\frac{p_{0}}{p}\right)^{n}\left(1-p^{2} / p_{\max }^{2}\right)^{-(3-n) / 2}
$$

where $p$ is the particle momentum, $N_{0}$ and $p_{0}$ set the absolute number of particles, $p_{\max }=E_{\max } / c$ is the maximum momentum determined by IC losses (eq. 1), and $n=(\chi+2) /(\chi-1)$. For a strong shock $\chi=4$; the resulting particle spectrum is a $p^{-2}$ power law with a weak spike at the high energy cutoff. After the particles leave the shock, further IC cooling causes the upper and lower limits of the power law to slide toward lower energies, but the $n=2$ slope is unchanged.

\subsection{InVERSE Compton COOLING OF THERMAL ELECTRONS}

IC scattering can also be the most important cooling process for the thermal electrons in colliding wind shocks (Cherepashchuk 1976). For a gas of temperature $T$ embedded in a radiation field of energy density $U_{p h}$, the cooling rate is

$$
J=4 \frac{\sigma_{T}}{m_{e} c} n_{e} U_{p h} k T
$$

where $n_{e}$ is the electron number density. The collisional cooling rate is $\Lambda_{\text {coll }}(T) n_{e} n$ where $\Lambda_{\text {coll }}$ is the cooling coefficient and $n=\rho / m_{H}$. In a stellar wind both the number density $n$ and $U_{p h}$ vary as $r^{-2}$; consequently, we can derive an effective cooling coefficient for IC scattering:

$$
\Lambda_{I C}=\frac{4 \sigma_{T} m_{H} L v_{\infty} k T}{m_{e} c^{2} 4 \dot{M}}=3.7 \times 10^{-23} \mathrm{ergs} \mathrm{cm}{ }^{3} / \mathrm{s} \frac{L_{6} T_{7} v_{\infty, 8.5}}{\dot{M}_{-6}}
$$

where $T_{7}=T / 10^{7} \mathrm{~K}, v_{\infty, 8.5} 10^{3.5} \mathrm{~km} / \mathrm{s}$ is the wind terminal velocity, and $\dot{M}_{-6} 10^{-6} \mathrm{M}_{\odot} / \mathrm{yr}$ is the wind mass loss rate. (The factor of 4 is included in the denominator to account for the compression of the wind in a strong shock.) Note that in a WR+O binary one typically should use both $L$ and $\dot{M}$ for the $\mathrm{O}$ star wind in this cooling calculation. The shock is usually much closer to the $\mathbf{O}$ star and so its luminosity dominates.

For a luminous star the IC cooling is stronger than collisional cooling when the temperature exceeds about $10^{7} \mathrm{~K}$. This is important when the shock is radiative, since IC cooling effectively steals energy from the shocked gas, reducing the energy available for thermal X-ray emission (Cherepashchuk 1976). This probably explains why Stevens, Blondin \& Pollock (1992) found that their model for V444 Cygni predicted too much X-ray emission: their neglect of IC cooling could easily lead to the factor of 10 overestimate of 
thermal X-ray emission from the shocked gas. The additional cooling can also change the shock from adiabatic to radiative, particularly for high velocity shocks $(v \sim 3000 \mathrm{~km} / \mathrm{s})$ where $\Lambda_{I C} \gg \Lambda_{\text {coll }}$.

\subsection{NON-THERMAL EMISSION MECHANISMS}

The major non-thermal emission mechanisms for both single stars and colliding wind binaries are synchrotron emission in the radio, inverse Compton emission from $\mathrm{X}$-ray to $\gamma$-ray energies, and pion decay high-energy $\gamma$-ray emission. White \& Chen (1992) discuss $\pi^{0}$ decay $\gamma$-rays from 0 stars; it typically produces $\gamma$-rays in the $50 \mathrm{MeV}$ to $10 \mathrm{GeV}$ energy range. We will not consider it further in this paper.

Synchrotron and inverse Compton emission have exactly the same function dependence on the particle energy distribution. Thus, in the absence of absorption there is a very close relationship between the radio synchrotron spectrum and the inverse Compton X-ray spectrum. For either process the total energy lost by a particle of energy $\gamma m_{e} c^{2}$ is

$$
P=4 \sigma_{T} c U \gamma^{2} / 3,
$$

where $U$ is the photon energy density (for IC emission) or the magnetic field energy density (for synchrotron emission.) The ratio of the synchrotron luminosity to the IC luminosity is then

$$
\frac{L_{s y n}}{L_{I C}}=\frac{U_{B}}{U_{p h}}=8.4 \times 10^{-4} \frac{B^{2} r_{A U}^{2}}{L_{6}} .
$$

Far out in the wind the magnetic field is azimuthal and depends on the surface magnetic field $B_{*}$, the stellar radius $R$, and the stellar rotation velocity $v_{\text {rot }}: B=B_{*}\left(v_{\text {rot }} R / v_{\infty} r\right)$ (Weber \& Davis 1967). In this region of the wind the $r$ dependence of $U_{B}$ and $U_{p h}$ cancel and we have

$$
\frac{L_{s y n}}{L_{I C}}=7.1 \times 10^{-4} \frac{1}{L_{6}}\left(\frac{B_{*, 100} R_{25} v_{r o t, 250}}{v_{\infty, 8.5}}\right)^{2}
$$

where $B_{*}=100 \mathrm{G} B_{*, 100}, R=25 \mathrm{R}_{\odot} R_{25}$, and $v_{\text {rot }}=250 \mathrm{~km} / \mathrm{s} v_{\text {rot }, 250}$. For typical values of these parameters in colliding winds we see that the IC luminosity is much larger than the synchrotron luminosity.

IC emission from a black-body radiation spectrum of temperature $T_{r}$ produces photons having the characteristic energy

$$
E_{I C}=5 k T_{r} \gamma^{2}
$$

while synchrotron photons have characteristic frequencies

$$
\nu_{s y n}=0.44 \nu_{B} \gamma^{2}
$$


where

$$
\nu_{B}=e B / 2 \pi m_{e} c=2.8 \times 10^{6} \mathrm{~Hz} B .
$$

If the electron spectrum is a power law of index $n$ (see eq. 3), then both the synchrotron and IC spectra have index $\alpha=(n-1) / 2$, where $L_{\nu} \propto \nu^{-\alpha}$. The luminosity ratio of the two spectra is given in eq. (8), and the shift in energy is

$$
E_{I C}=3.5 \mathrm{keV} \frac{\nu_{G H z} T_{r, 4}}{B}
$$

where $\nu_{G H z}$ is the synchrotron frequency in $\mathrm{GHz}$ and $T_{r}=10^{4} \mathrm{~K} T_{r, 4}$.

\subsection{ABSORPTION MECHANISMS}

The observed luminosities in these systems are reduced by absorption in both the radio and in soft $\mathrm{X}$-rays $(E \lesssim 1 \mathrm{keV})$. The $\mathrm{X}$-ray absorption is due to K-shell ionization of heavy elements; it does not significantly affect the $\mathrm{X}$-rays at the high energies considered here. The radio absorption is due to free-free absorption in the stellar winds and is of great importance. Figure 6 of Becker \& White (1994) shows the regions of the winds that are blocked by free-free absorption for HD 193793; so little of the wind interaction region is visible that Becker \& White conclude the spherically symmetric model for this system cannot explain the radio observations.

\subsection{Synchrotron SUPPRESSION VIA the RAZIN EFfECT}

Previous studies have neglected an important physical process: in many cases of interest, the Razin effect can suppress $\mathrm{GHz}$-frequency radio emission. The significance of this was first pointed out by Chen in his thesis (Chen 1992; Chen \& White 1994). The Razin effect effectively shuts off synchrotron emission below a critical frequency that depends on the ratio of the electron density to the magnetic field:

$$
\nu_{R}=20\left(n_{e} / B \sin \psi\right)=0.85 \mathrm{GHz} \dot{M}_{-6} /\left(r_{A U}^{2} v_{\infty, 8.5} B \sin \psi\right)
$$

where $\psi$ is the angle between the electron's velocity and the magnetic field. For single stars, the Razin effect rules out models with weak surface magnetic fields (Chen 1992; Chen \& White 1994). For plausible values of the magnetic fields in colliding winds, radio emission at the frequencies usually observed (1.5-15 GHz) can be suppressed. For example, in the colliding wind binary HD 193793, if we assume a surface magnetic field on the WR star of $500 \mathrm{G}$, we find that the Razin frequency in the shocked WR wind is greater than $7 \mathrm{GHz}$ throughout the orbital period (Fig. 2). Thus, the WR star cannot contribute to the observed radio emission unless its surface magnetic field is extraordinarily high $(5000 \mathrm{G})$. The $\mathrm{O}$ star has a somewhat lower Razin frequency, but it too can suffer Razin suppression. 


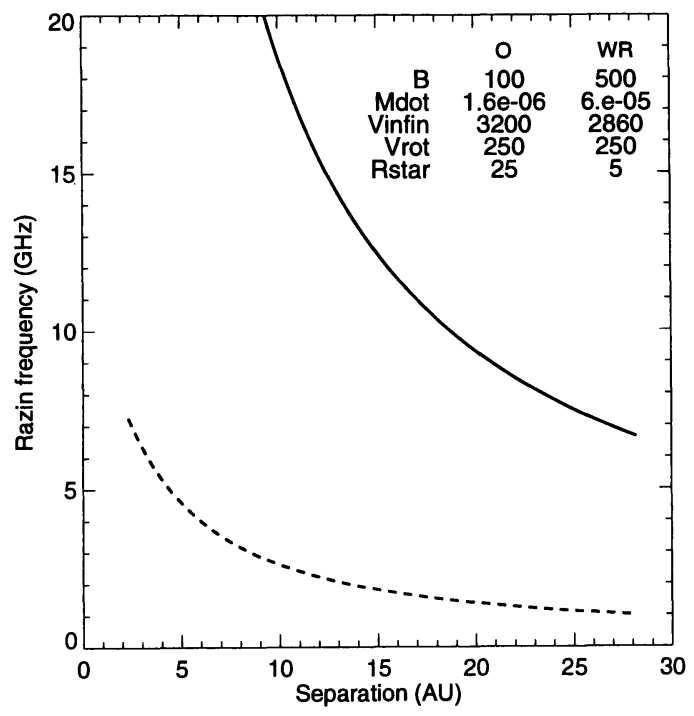

Fig. 2. Razin frequencies as a function of separation for $O$ (dashed line) and WR (solid line) colliding winds in the binary system HD 193793. The compression of the wind and the magnetic field by the shock are included. Synchrotron radio emission is suppressed for frequencies below $\nu_{R}$.

\section{Binaries versus single stars: differences}

There are many more similarities than differences between the physics of non-thermal emission in single stars and in colliding wind binaries. Nonetheless, there are some significant differences. First, the shocks in colliding wind binaries are stronger than those in unstable winds, and because the shocks often occur very far out in the winds, they are often adiabatic. Shocks in single $\mathbf{O}$ star winds are almost always isothermal. Second, colliding winds have considerably simpler (and better understood) geometries, with only a single strong shock in the wind rather than the chaotic jumble of weaker shocks found in single star winds. This simpler geometry is especially important for understanding non-thermal radio emission, because free-free absorption by the stellar winds has a major effect on the observable synchrotron spectrum. Third, in single star winds the high energy emission is dominated by particles accelerated close to the star, while the synchrotron radio emission is produced by particles accelerated well out in the wind (Chen \& White 1994). As a result, in binaries the same electrons emit both observable X-rays and the observed radio emission. In single stars these emissions come from distinct regions and so the correlation expected between the luminosities in the $\mathrm{X}$-ray and the radio is much less clear. 


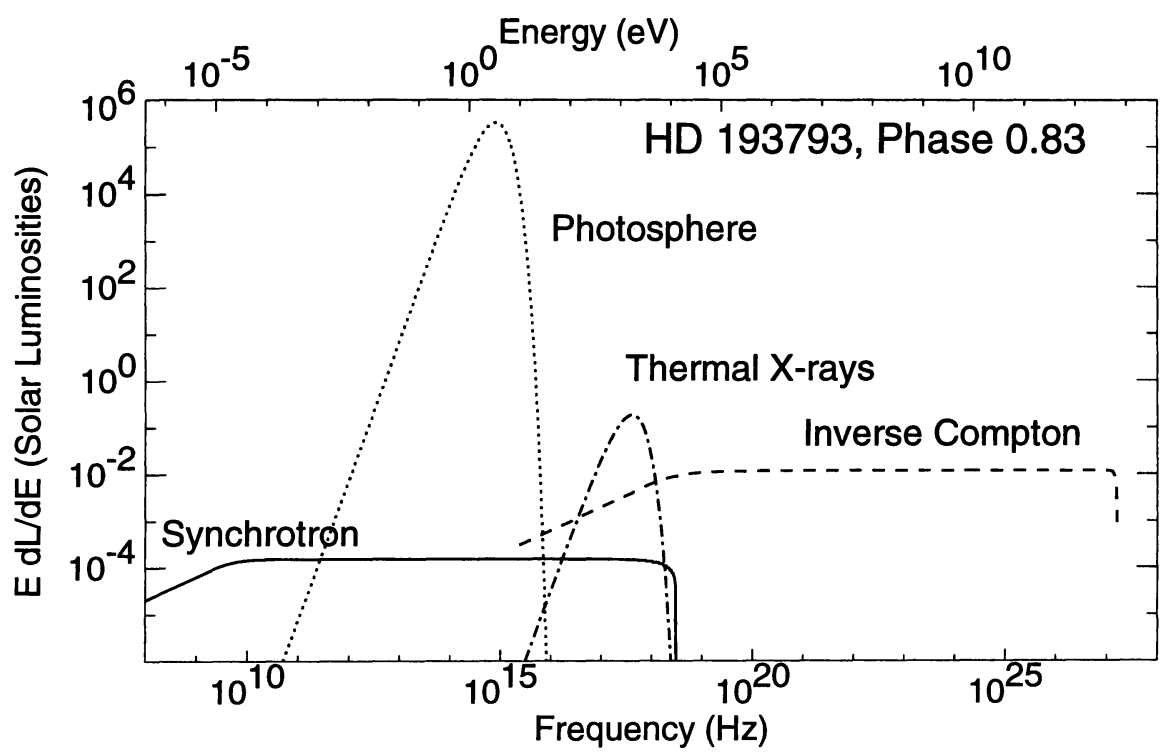

Fig. 3. Computed spectrum of HD 193793 from radio to $\gamma$-ray energies. Photospheric and thermal X-ray emission are indicated only schematically. The normalization of the synchrotron emission was set from the observed radio emission (Becker \& White 1994); the shape of the spectrum was not a free parameter. Free-free absorption of the radio is not included. The normalization of the predicted high-energy inverse Compton emission is then determined from the synchrotron flux density. A decrease in the $O$ star magnetic field (assumed to be $100 \mathrm{G}$ at the stellar surface) would lead to an increase in the inverse Compton luminosity but would also result in Razin suppression of the low frequency radio emission.

\section{HD 193793}

We have applied these calculations to the $\mathrm{WR}+\mathrm{O}$ colliding wind system HD 193793. This system is a long period (7.9 yr) binary with a highly eccentric orbit $(e=0.84)$. We use the stellar parameters as determined by Williams et al. (1990) and van der Hucht $(1991,1992)$. Our results are shown in Figure 3. The most interesting point of the above calculation is that there is a direct correlation between the synchrotron radio emission and inverse Compton X-ray and $\gamma$-ray emission. We have computed the synchrotron and inverse Compton spectra at phase 0.83 , when the $20 \mathrm{~cm}$ emission is at a maximum (Becker \& White 1994), assuming a surface magnetic field on the $\mathrm{O}$ star of $100 \mathrm{G}$. There is considerable uncertainty about the efficiency with which electrons are accelerated by shocks; however, we can use the observed radio flux density to normalize both the synchrotron and IC spectra. The predicted radio spectrum in the absence of absorption is $F_{\nu} \propto \nu^{-0.5}$ below $\nu=10 \mathrm{GHz}$ and $F_{\nu} \propto \nu^{-1}$ at higher frequencies. The high frequency cutoff 
is set by the maximum energy of particle acceleration $E_{\max }$ (eq. 1) and is at the astoundingly high energy of $10 \mathrm{keV}$. Free-free absorption has not been included in the spectrum shown in Figure 3, but that should not affect the normalization because we used the $15 \mathrm{GHz}$ flux density measurements from Becker \& White, which are not much affected by absorption.

The high energy synchrotron emission is not observable, though, because inverse Compton emission is much stronger. The IC spectrum has exactly the same shape as the synchrotron spectrum $\left(E^{-1 / 2}\right.$ breaking to $E^{-1}$ at about $15 \mathrm{keV}$ ) but is more luminous by a factor of 70 . The high energy cutoff of the IC spectrum is at about $1000 \mathrm{GeV}$. Unfortunately, this is such a high energy that the considerable luminosity in IC emission $\left(1 \times 10^{33} \mathrm{ergs} / \mathrm{s}\right)$ is spread over a very wide energy range and so will be quite difficult to detect. The best hope for detection of this emission is probably around 10-20 keV, where the thermal X-ray emission has decreased to the point that the underlying non-thermal power-law may become visible.

A lower surface magnetic field would reduce the high energy cutoff of both the synchrotron and IC spectra; however, if the surface magnetic field were much smaller than $100 \mathrm{G}$ then the Razin effect would completely suppress the (observed) 1-10 GHz emission. In fact, the Razin effect is already suppressing any radio emission from the WR wind shock. Presumably the WR shock accelerates electrons at about the same rate as the $\mathrm{O}$ shock (both the velocities and densities are similar), so we have multiplied our estimate of the IC luminosity by 2 to compensate for these "non-synchrotron" electrons.

\section{Conclusions}

Despite the differences between single stars and colliding wind systems, there are close similarities between them. Generally the physical effects we have found to be important in shock-permeated winds are also important in colliding wind shocks.

A major advantage of colliding wind binaries is that a direct connection may be made between the observed non-thermal radio emission and non-thermal X-ray emission in colliding wind binaries because the same relativistic electrons lead to both types of emission. If we can measure the luminosities from both of these processes, we can get a direct measurement of the magnetic field in the vicinity of the shock. Once the magnetic field (and other wind parameters) has been specified, the spectra of both radio synchrotron emission and X-ray $/ \gamma$-ray inverse Compton emission are completely determined.

Two important physical effects appear to have been ignored in previous work. Inverse Compton losses are important not only for relativistic particles but also for the hot, shocked gas. For low velocity shocks $(v \sim 500 \mathrm{~km} / \mathrm{s}$, $\left.T \sim 3 \times 10^{6} \mathrm{~K}\right)$ the usual collisional cooling processes dominate, but for 
higher velocities $\left(v>1000 \mathrm{~km} / \mathrm{s}, T>1 \times 10^{7} \mathrm{~K}\right)$ inverse Compton losses are often stronger than collisional cooling. An important consequence of this is that the thermal X-ray emission will be much reduced in systems with radiative shocks such as V444 Cygni (Cherepashchuk 1976). This probably explains why the model of Stevens, Blondin \& Pollock (1992) predicts more $\mathrm{X}$-rays than are observed from V444 Cygni.

The Razin effect suppresses low-frequency synchrotron radio emission when the magnetic field is not high. In $\mathrm{O}+\mathrm{WR}$ colliding wind systems, this typically means that particles accelerated in the WR wind shock cannot produce the observed radio emission, and emission from the the $\mathrm{O}$ wind shock may also be suppressed in some cases. The Razin effect can be used to rule out models with low magnetic fields, because they cannot produce the observed $\mathrm{GHz}$ radio emission regardless of the efficiency of electron acceleration.

\section{References}

Abbott, D.C., Bieging, J.H., Churchwell, E.B. 1984, $A p J$ 280, 671

Abbott, D.C., Bieging, J.H., Churchwell, E.B. 1985, in: R.M. Hjellming \& D.M. Gibson (eds.), Radio Stars, (Dordrecht: Reidel), p. 219

Becker, R.H., White, R.L. 1985, ApJ 297, 649

Becker, R.H., White, R.L. 1994, these proceedings

Cassinelli, J.P., Swank, J.H. 1983, ApJ 271, 681

Chen, W. 1992, PhD thesis, Johns Hopkins University

Chen, W., White, R.L. 1991a, ApJ 366, 512

Chen, W., White, R.L. 1991b, ApJ (Letters) 381, L64

Chen, W., White, R.L. 1994, in: A.F.J. Moffat \& A. Fullerton (eds.), Instability and Variability of Hot Star Winds, in press

Cherepashchuk, A.M. 1976, Sov. Astron. (Letters) 2, 138

Eichler, D., Usov, V. 1993, ApJ 402, 271

Luo, D., McCray, R., Mac Low, M.-M. 1990, ApJ 362, 267

Montmerle, T. 1990, in: K. A. van der Hucht \& B. Hidayat (eds.), Wolf-Rayet Stars and Interrelations with other Massive Stars in Galaxies, Proc. IAU Symp. No. 143 (Kluwer: Dordrecht), p. 397

Pollock, A.M.T. 1987, $A \& A$ 171, 135

Pollock, A.M.T. 1991, in: K. A. van der Hucht \& B. Hidayat (eds.), Wolf-Rayet Stars and Interrelations with other Massive Stars in Galaxies, Proc. IAU Symp. No. 143 (Kluwer: Dordrecht), p. 102

Stevens, I.R., Blondin, J.M., Pollock, A.M.T. 1992, ApJ 386, 265

van der Hucht, K.A. 1991, in: K. A. van der Hucht \& B. Hidayat (eds.), Wolf-Rayet Stars and Interrelations with other Massive Stars in Galaxies, Proc. IAU Symp. No. 143 (Kluwer: Dordrecht), p. 19

van der Hucht, K. A. 1992, in: L. Drissen, C. Leitherer, A. Nota (eds), Nonisotropic and Variable Outflows from Stars, ASP Conf. Series 22, p. 253

Weber, E.J., Davis, L. 1967, $A p J$ 148, 217

White, R.L. 1985, $A p J$ 289, 698

White, R.L., Becker, R.H. 1982, ApJ 262, 657

White, R.L., Chen, W. 1992, ApJ (Letters) 387, L81

Williams, P.M., van der Hucht, K.A., Pollock, A.M.T., Florkowski, D.R., van der Woerd, H., Wamsteker, W.M. 1990, MNRAS 243, 662 


\section{DISCUSSION:}

Nussbaumer: What kind of radiation field is necessary for inverse Compton cooling to become effective?

White: The inverse Compton cooling is proportional to the gas temperature and the stellar luminosity. When the effective cooling coefficient drops below $\sim 10^{-23}$ at $\mathrm{T}=10^{8}$ it will probably not be very important. This typically occurs at $\mathrm{L} \sim 10^{4} \mathrm{~L}_{\circ}$ in a wind with $\mathrm{V}_{\infty} \sim 3000$ $\mathrm{km} / \mathrm{s}$.

Usov: As it is shown by Cherepashchuk the gas cooling by inverse Compton is essential for close binaries, like V444 Cyg, only. It is my remark. My question is what is the highest energy of $\gamma$-rays which may be observed from WR 140 with taking into electron-position pair creation on UV photons?

White: Thank you for pointing out the Cherepashchuk work - I am not surprised to find out this effect has been recognised before, but it has been ignored in recent years.

The remark is correct that for wide binaries such as WR 140, neither inverse Compton nor collisional cooling has much affect on the shocked gas. The shocks in such binaries are adiabatic. On the other hand, even in those stars high velocity shocked gas loses more energy via inverse Compton cooling than via Bremsstrahling and other collisional processes.

It is quite possible that the very highest energy $\gamma$-rays predicted by this model $\left(\mathrm{E} \sim 10^{12} \mathrm{eV}\right)$ may be involved in other energetic processes. I do not know the details of such processes.

Pollock: Are any of the non-thermal radio stars NOT binaries?

White: Certainly many of the $\mathrm{O}$ stars with non-thermal radio emission are not known to be binaries, though it is not easy to detect companions for such luminous stars. Abbott et al. concluded that the frequency of non-thermal, radio emission among $\mathrm{O}$ stars was too high for all the non-thermal sources to be binaries. Also, Chen (1992) showed that a large fraction of luminous stars with high terminal velocities are non-thermal radio sources; this argues against a binary origin.

I think there are strong statistical arguments against the proposal that all non-thermal $\mathrm{O}$ stars are binaries though any individual object could be discovered to be a binary.

van der Hucht: To my knowledge, all hot massive non-thermal radio stars which have been observed more than once appear to be variable in their non-thermal radio emission. Can your single-star model produce variable non-thermal radio emission?

White: The predicted non-thermal emission does have strong dependences on some parameters including the magnetic field. Possibly small variations in these parameters could lead to the observed variability.

On the other hand, the striking lack of variability in X-ray emission discussed by Pollock indicates that the global structure of shocks in the wind does not vary much. Models that rely on shock fluctuations to get radio variability may not work.

Another possibility is that only the very strongest shocks are able to reach the radio-emitting region (at several hundred stellar radii). Even if the bulk of the X-ray shocks do not vary much, the number of very strong shocks might undergo large fluctuations, leading to the observed synchrotron variability.

Owocki: The need to accelerate particles in multiple shocks at $>100 \mathrm{R}^{*}$ in single stars presents a real problem, since I doubt the wind instability shocks will persist that far out. As for binaries, how is it possible to achieve the required acceleration in a single shock, when you always spoke in terms of multiple shocks in single stars? 
White: The need for shocks far out in the wind is certainly a problem, though their existence seems unavoidable based on the radio observations. They may not be very strong shocks (100 $\mathrm{km} / \mathrm{s})$.

For electrons, multiple shock acceleration is not so important in Chen's models because inverse Compton losses cause the electrons to lose almost all their energy between shocks. They must be re-accelerated at each shock anyway. The total pressure in relativistic electrons may be higher as a result of multiple shock acceleration.

Acceleration of ions is more strongly affected by multiple acceleration episodes because ions do not lose their energy after acceleration. There might be observable difference between the pion-decay $\gamma$-ray spectra for multiple and single shock acceleration (if such $\gamma$-rays are observed!).

Hill: Do you assume a dipole magnetic field for the $\mathrm{O}$ star?

White: The magnetic field model is from Weber \& Davis. It is dipolar very close to the star but at large radii is tangential and drops as $1 / \mathrm{r}$. The magnetic field is weak compared to other forces and so is dragged away from the star by the stellar wind.

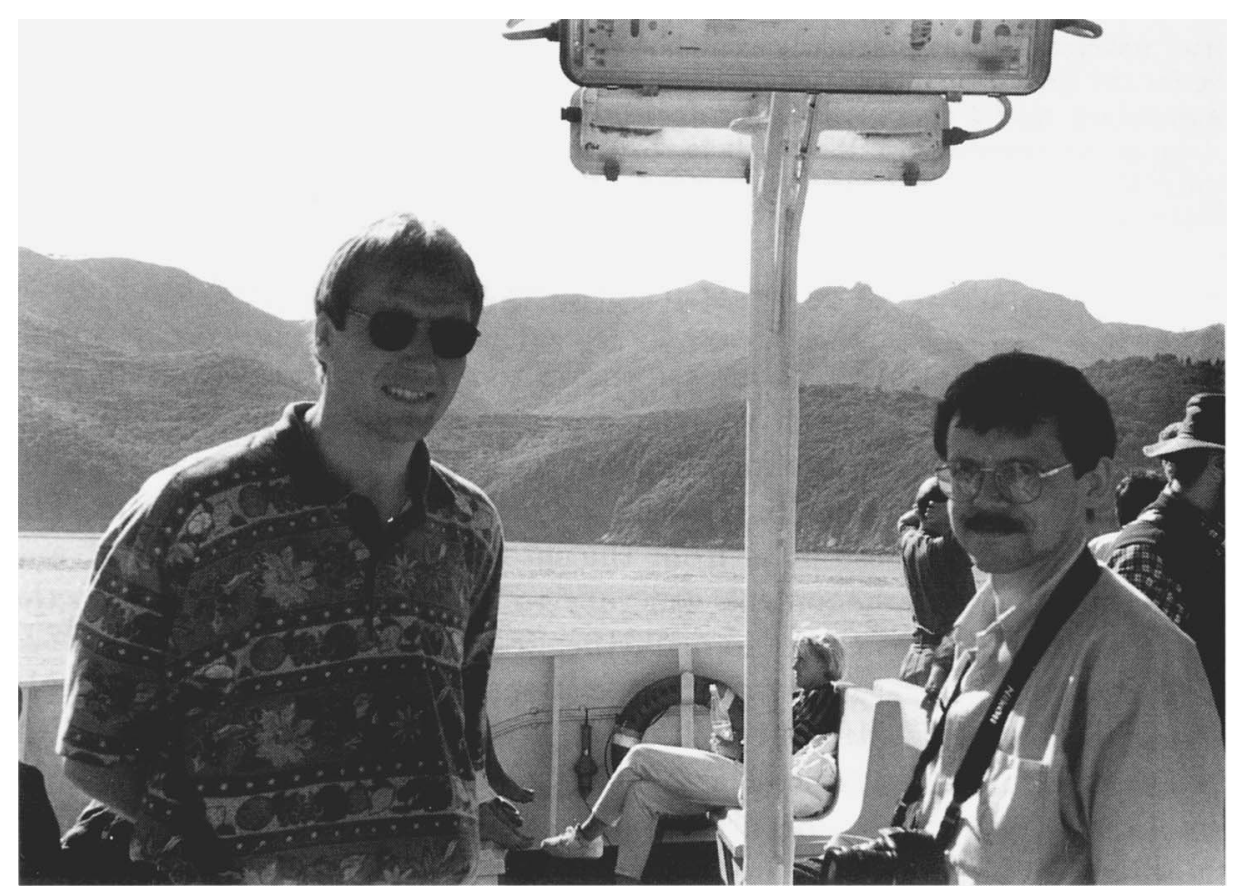

Kaper, Antokhin 